\title{
Kaija Saariaho, compositrice, ou quand devient-on un compositeur classique?
}

\section{Henri-Claude Fantapié}

\section{OpenEdition}

1 Journals

\section{Édition électronique}

URL : https://journals.openedition.org/efo/2585

DOI : $10.4000 /$ efo. 2585

ISSN : 2275-1947

Éditeur

INALCO

Édition imprimée

Date de publication : 1 décembre 2013

ISBN : 978-2-343-04446-0

ISSN : 0071-2051

\section{Référence électronique}

Henri-Claude Fantapié, « Kaija Saariaho, compositrice, ou quand devient-on un

compositeur classique? », Études finno-ougriennes [En ligne], 45 | 2013, mis en ligne le 12 février 2015, consulté le 08 juillet 2021. URL : http://journals.openedition.org/efo/2585 ; DOI : https://doi.org/ $10.4000 /$ efo. 2585

Ce document a été généré automatiquement le 8 juillet 2021.

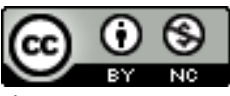

Études finno-ougriennes est mis à disposition selon les termes de la Licence Creative Commons Attribution - Pas d'Utilisation Commerciale 4.0 International. 


\title{
Kaija Saariaho, compositrice, ou quand devient-on un compositeur classique?
}

\author{
Henri-Claude Fantapié
}

1 La série de concerts organisés du 17 au 23 avril 2013 par la Cité de la musique, sous l'appellation "Domaine privé », autour de la compositrice Kaija Saariaho et de son œuvre, a été un événement important de la vie musicale française, plus particulièrement parisienne et franco-finlandaise. L'événement a commencé par une soirée de présentation à l'Ambassade de Finlande, partie prenante de l'organisation, prélude à une soirée de ballets, un concert de musique de chambre, deux concerts symphoniques et un forum. Cet hommage à une grande dame de la composition a permis d'entendre un éventail de ses œuvres et quelques-unes de ses références musicales, probablement parmi les plus marquantes.

2 Il est toujours un peu impressionnant pour un créateur d'être ainsi le centre d'un tel événement et d'y participer physiquement. Si Kaija Saariaho figure parmi les compositeurs actuels les plus importants, son caractère réservé ne la pousse pas naturellement à se mettre en avant. Le ton a été donné le mardi 16 avril lors de la soirée sur invitation à l'Ambassade de Finlande, quand son ami le violoncelliste a interrogé Kaija sur elle-même, sur son œuvre et sur l'événement. À une de ses questions, la compositrice répondit par une autre interrogation tout à fait personnelle : quand a-t-on l'impression de devenir un "classique »? Cela fait longtemps, en effet, que Saariaho compose, accompagnée d'un certain nombre d'amis et complices qui, à l'instar d'Anssi Karttunen, partagent avec elle les œuvres qu'elle écrit (et qu'elle leur destine souvent). À l'origine, en Finlande, il y eut un groupe de jeunes compositeurs qui avait pris le nom de Korvat auki! (Ouvrez les oreilles!), au nombre desquels on comptait le chef d'orchestre Esa Pekka Salonen (né en 1958), les compositeurs Magnus Lindberg, Olli Kortekangas, Jukka Tiensuu et Eero Hämeenniemi. Autour d'eux, on retrouvait de jeunes instrumentistes, dont le violoncelliste Anssi Karttunen (né en 1960) déjà cité, la flûtiste Camilla Hoitenga, la pianiste Tuija Hakkila, les cantatrices Pia Freund et Anu 
Komsi. Plus tard, à Paris, une longue collaboration s'est établie à l'IRCAM avec l'artiste multimédia Jean-Baptiste Barrière, puis avec l'écrivain Amin Maalouf. J'interromps cette énumération au moment où, au-delà des frontières de ses pays de naissance et d'adoption, les œuvres de Kaija ont attiré d'autres artistes et metteurs en scène fidèles, comme Dawn Upshaw, Emmanuel Ax, Peter Sellars, Barbara Hannigan et les Finlandais Sakari Oramo ou Karita Mattila. Mais arrêtons là, pour rester sur la génération de ceux qui ont étudié ensemble, se sont fréquentés, ont évolué au milieu des mêmes événements culturels et sociétaux. Aujourd'hui, ce sont eux qui délivrent leur enseignement à une nouvelle génération, tandis que leurs interprétations des compositeurs qui sont leurs contemporains deviennent des références. Un des intérêts de cette semaine de concerts a justement été de réunir ces différentes générations d'interprètes et de découvrir l'apport des plus jeunes d'entre eux dans une musique parfois composée avant leur naissance, ou dans un autre pays que le leur. À son grand étonnement, au cours des répétitions, Saariaho, qui rencontrait pour la première fois certains de ces jeunes interprètes, a découvert qu'il n'y avait aucune rupture transgénérationnelle dans la compréhension de son œuvre. J'ajouterai que la même remarque est valable avec des interprètes de pays, voire de cultures, très différents. Pour rester sur ce seul sujet, n'est-ce point parce qu'elle est maintenant un compositeur «classique », qui concrétise son intégration dans l'histoire musicale, audelà des modes et des péripéties de notre époque ?

Cette soirée privée à l'Ambassade de Finlande a permis d'entendre l'ensemble New Yorkais ICE (International Contemporary Ensemble) dans des œuvres qui suggérèrent à Pierre Gervasoni, dans Le Monde du jeudi 18 avril, le titre de son article : «Les caresses telluriques de Kaija Saariaho ». Le répertoire joué partait de pièces de 1991 (Fall from Maa) pour aller jusqu'à une création française (Duft, de 2012). L'interprétation était celle d'un ensemble d'une exceptionnelle qualité, à l'image de Claire Chase, flûtiste extraordinairement engagée musicalement et techniquement.

Nous avons ensuite rejoint la Cité de la musique pour la suite du programme.

5 Le 17 avril je n'ai pu ni entendre ni voir le ballet Maa (Terre, 1991), en création française, par les musiciens du même ensemble que la veille. Le 18, ce sont les amisinterprètes qui sont intervenus, avec les Sept Papillons pour violoncelle de 2000 (par Anssi Karttunen), Serenatas de 2008 pour piano, violoncelle et percussions, le quatuor à cordes Terra memoria (de 2007-2009) et après le Quatuor à cordes de Jean Sibelius, Mirage, de 2007-2010, dans une version en trio par Anssi Karttunen, Tuija Hakkila et la soprano Pia Freund.

6 Le 19 avril ont été données, au cours d'un concert de l'Orchestre philharmonique de Radio France, trois créations françaises: Asteroid 4179: Toutatis (2006), composé pour l'Orchestre philharmonique de Berlin dans le cadre d'un projet autour de Planets de Gustav Holst, Laterna magica (2008), inspiré par le livre de mémoires du cinéaste suédois Ingmar Bergman, et les Leino songs (2000-2007), quatre mélodies sur des poèmes d'Eino Leino (1878-1926). Ces pièces étaient confrontées à deux œuvres centenaires de Jean Sibelius, elles-mêmes fort différentes l'une de l'autre : Luonnotar op. 70 (1913) et la Symphonie $\mathrm{n}^{\circ} 7$ op. 105 (1914-1915-1924).

7 Le 20 avril, un forum autour de l'opéra Adriana Mater comprenait, outre quatre œuvres de musique de chambre de Kaija (Cendres, Vent nocturne, Dolce tormento, Je sens un deuxième cour), l'audition des deux sonates de 1915, pour violoncelle et piano et pour 
flûte, alto et harpe de Claude Debussy, avec la participation de musiciens de l'Ensemble Intercontemporain.

Le mardi 23, le dernier concert, donné par l'ensemble finlandais Avanti!, était un des plus originaux par son programme qui, en première partie, faisait alterner trois mélodies de Jean Sibelius avec une œuvre de Paavo Heininen (né en 1938), qui fut le professeur de Kaija Saariaho à l'Académie Sibelius - la Musique d'été de 1963-1967, œuvre qui fut considérée comme particulièrement moderniste dans la Finlande de l'époque - et une pièce de Lotta Wennakoski de 2005 : Kuule II (Écoutez II). La deuxième partie du concert permit d'entendre Emilie suite, tirée en 2011 de l'opéra sur la vie de l'amie de Voltaire et de Saint-Lambert, Émilie du Châtelet.

Disons, et ce n'est pas sans importance, que les interprètes que nous avons entendus dans ces différents concerts ont parfaitement justifié la remarque liminaire de Saariaho et montré que les plus jeunes d'entre eux étaient tout aussi familiers de son style que leurs plus illustres aînés, qu'ils soient finlandais comme l'étonnant et explosif chef d'orchestre Santtu-Matias Rouvali (qui, d'une certaine manière, m'a rappelé l'effet que me fit Seiji Ozawa quand je l'ai vu pour la première fois au pupitre dans la classe de direction d'Eugène Bigot), ou comme la flûtiste Claire Chase, tout aussi explosive et concentrée à la fois, tous deux s'insérant naturellement dans la lignée des Freund, Komsi, Karttunen, Hakkila, Hoitenga et autres Hannigan, mais appartenant visiblement à une nouvelle génération d'interprètes qui, nés et formés dans l'environnement de musiques que leurs aînés avaient découvertes comme un monde nouveau et inconnu, les considéraient comme étant un langage naturel pour eux, déjà "classique », peutêtre même plus aisé à pénétrer, comprendre et défendre.

\section{L'œuvre}

Plus qu'encyclopédique, voire didactique, dans le choix des ouvrages, cette série de concerts a plutôt été une succession de coups de cœur musicaux de la compositrice. La sélection était toutefois suffisamment bien faite, qualitativement et quantitativement, pour qu'on puisse en tirer un triple enseignement.

11 Le premier, on l'a vu, permet de penser qu'il existe maintenant ce qu'on pourrait appeler un style d'interprétation (on pourrait presque dire qu'on assiste à la naissance d'une tradition interprétative, si ce mot n'avait pas une connotation aussi négative) de l'œuvre de la compositrice. On ne peut que comparer le rôle que Kaija joue avec ses interprètes à l'importance de celui tenu par Henri Dutilleux préparant l'exécution de ses œuvres, lui qui donnait un enseignement précieux pour les interprètes présents et futurs, sans pourtant jamais les emprisonner dans des contraintes desséchantes. Il a été intéressant de noter que, outre la confrontation entre plusieurs générations d'interprètes, entre " anciens » et " nouveaux », on a également pu établir un parallèle entre musiciens finlandais, français et américains. La musique de Saariaho n'est pas une musique nationale, elle appartient à des interprètes de toutes origines, donc aussi à tous les publics.

12 Le second enseignement permet de situer Saariaho dans le temps. Les références qu'elle a choisies parmi les compositeurs d'hier et d'aujourd'hui ont été peu nombreuses et d'autant plus éclairantes. Parmi les "pères », il y eut Debussy et surtout Sibelius. On peut s'interroger sur les choix d'œuvres du premier (les Sonates « françaises » de la fin de la vie), beaucoup moins dans le cas de Sibelius: la comparaison entre le style et 
l'expression de l'œuvre vocale de la compositrice avec ceux des ponctuations lyriques des trois mélodies de Sibelius dans le concert du 23 nous a contraints à renouveler notre écoute de ces œuvres et à rapprocher deux modes d'expression qui, loin de s'affronter, comportaient des points de convergence. À côté de ces deux géants, les auditeurs français ont aussi pu découvrir deux compositeurs peu joués chez nous: Paavo Heininen (né en 1938), qui, auteur d'une œuvre de qualité, abondante et variée, compta parmi les compositeurs finlandais les plus radicaux de la fin des années cinquante et, à partir de 1966 fut, à l'Académie Sibelius, le professeur de la quasitotalité des compositeurs finlandais importants d'aujourd'hui, et Lotta Wennakoski (née en 1970) qui a elle-même été l'élève de Heininen, de Eero Hämeenniemi et de Saariah et qui partage avec cette dernière - faut-il encore l'évoquer - le fait d'être une compositrice. Il manquait peut-être à ce panorama une œuvre de style spectral de Gérard Grisey (1946-1998) ou/et de Tristan Murail (né en 1947), qui aurait parfaitement complété l'aperçu de l'environnement de la formation du langage de la compositrice, mais le cadre de ce " Domaine privé » ne permettait probablement pas d'aller beaucoup plus loin que ce qui était proposé.

Le troisième enseignement est que cette anthologie a permis de situer une œuvre en devenir, nell mezzo del cammin... (mais où la selva n'a rien d'obscur!), une œuvre protéiforme et pourtant d'une remarquable homogénéité. À l'origine, il y a le son, le timbre, la couleur et le spectre sonore, l'intimité du soliste et la musique de chambre, la nature et l'espace. Certains ajouteront : une vision féminine. Les partitions pour soliste ou formation de chambre ont dominé ; l'opéra a été, par la force des choses, un peu sacrifié ; le ballet, l'électroacoustique ont été présents. Reste que certaines œuvres pour soliste et orchestre ou pour orchestre seul se doivent d'être maintenant entendues par l'auditeur qui découvrait le monde de Saariaho. Je pense à Du cristal... à la fumée (1990) ou à D'Om le vrai sens (2010) pour clarinette et orchestre, pour ne prendre que ces deux exemples qui marquent une évolution stylistique et peut-être d'attitude, qu'on a pu ressentir dès le premier soir quand, après Sept papillons (2000), nous avons entendu l'explosif Terrestre $(2003)^{1}$ qui me semble présenter une évolution expressive sensible.

14 Si la musique de Saariaho ne présente pas de caractéristiques "nationales ", est-il possible d'évoquer des caractères «féminins » à son propos? Je ne me risquerai pas, dans cet article, à développer ce point. Partant du principe de l'auditeur est le recréateur final de l'œuvre, c'est à lui qu'il faut laisser le soin de conclure, en attendant, cet été, le festival « Voices of the century » à Kuhmo², largement ouvert aux œuvres de Saariaho et à de nouvelles compositions, dont il est trop tôt pour parler.

\section{NOTES}

1. Qui ne connaît pas ces œuvres peut les entendre sur Youtube par le violoncelliste Alexis Decharmes, en CD, et par Chase et ICE sur vimeo.com/48560029. Pour D'Om le vrai sens, on ne peut pas ignorer le CD qui comprend également Laterna Magica et les Leino songs, ces deux dernières œuvres données au cours de «Domaine privé », ici enregistrées par Kari Kriikku, clarinette, Anu Komsi, soprano et l'Orchestre de la Radio finlandaise, dirigé par Sakari Oramo, chez Ondine. 
2. et

INDEX

Index géographique : Finlande, Kuhmo, Paris

Mots-clés : académie Sibelius, musicologie, IRCAM, Korvat auki !, musique contemporaine Index chronologique : XXIe siècle

Keywords : Contemporary Music

Thèmes : musicologie 